\title{
The maximum melting temperatures in strain-crystallized van der Waals networks*)
}

\author{
B. Holl'), H.-G. Kilian' ${ }^{1}$ ), and G. S. Y. Yeh' ${ }^{2}$ ) \\ 1) Abteilung Experimentelle Physik, Universität Ulm, Oberer Eselsberg, Ulm, F. R. G., and \\ $\left.{ }^{2}\right)$ Department of Chemical Engineering, The University of Michigan, Ann Arbor, USA
}

\begin{abstract}
A theory of strain-crystallization of random networks comprised of stereoregular chains is developed. The crosslinks are assumed to be expelled from crystal cores. For this reason, the rubber is considered to be represented as a random eutectoid copolymer, the thermodynamics of strain crystallisation of which is described by the use of the van der Waals model of networks. The strain dependence of the maximum melting temperatures, the degree of crystallinity and the average thickness of the crystallites calculated are shown to be in fair accord with experimental data.
\end{abstract}

Key words: Strain induced crystallization, van der Waals network, eutectoid copolymer, maximum melting temperature, degree of crystallinity, crystal size.

\section{Introduction}

The attention of various authors has been directed to a concise interpretation of the strain-induced crystallization of rubber [1-9]. The first to develop the basic principles was P.J. Flory [1]. Related to the model of a Gaussian network, treated as a single component system, the basic phenomena of strain-induced crystallization were elucidated by his considerations. Gaylord improved the theory by taking into account finite chain lengths and the orientational distribution of the crystallites found in strained networks [2,3]. A discussion of melting temperatures at different elongations has also been given by Mandelkern [4], Krigbaum, Flory, Oth, and Gent [5-9]. However, one essential problem was not solved in all of these approaches: the finite degree of crystallinity and its well known dependence on temperature and strain [6-9] is a priori not described. In Flory's treatment for example, the single component system has to undergo a phase transition with the classical phenomenon of indifferent coexistence of oriented crystallites in the remaining non-

*) Dedicated Prof. Dr. R. Bonart on the occasion of his 60th birthday. crystallized network. The consequence is that crystallization is completed at constant intensive variables (constant $T, P$ and $f$ ), leading at the end to the fully crystallized system.

It is now of substantial significance to take into consideration that the crosslinks are in fact found to be expelled from the well ordered crystal cores [1-11]. The crosslinks distributed along the chains are thus expected to severely constrain the crystallization abilities within networks comprised of stereoregular chains. Strictly speaking, each network should preferably be considered as a "crosslinked eutectoid copolymer", the crystallization of which is regulated by its chemical structure. It has been shown that the melting process of PE networks with different degrees of crosslinking can be fully described on these lines $[10,11]$.

We found it interesting to prove whether this theory could be extended for treating strain-induced crystallization in networks. We were in the position to take advantage of the existing van der Waals concept by which a description of the thermoelastic properties of rubbers can be made quantitative within the total range of strains [12-14]. We present in this paper first considerations on these lines by which we are enabled to describe the maximum melting temperatures of rubber in dependence on the degree of crosslinking and on the 
state of strain. Moreover, having developed a theory which delivers a complete description of crystallized equilibrium states in isotropic and in strained networks, it is decisive for its value to prove whether essential structure parameters in the oriented semicrystalline system (average thickness of the crystallites and mass fraction of the crystallites) will as well be computed in agreement with experimental data $[10$, 11].

\section{The van der Waals network [12]}

The strain-energy function of a van der Waals network, $W(\lambda)$ can be written as

$$
\begin{aligned}
W(\lambda)= & \frac{G_{o} T}{\lambda_{m}^{2} M_{S t}}\left\{-\left(\ln \left(1-\eta^{1 / 2}\right)+\eta^{1 / 2}\right) 2 \phi_{m}\right. \\
& \left.-\frac{2}{3} a \phi^{3 / 2}\right\}
\end{aligned}
$$

where $G_{o}$ is a constant, $T$ the absolute temperature, $M_{S t}$ the molecular weight of the stretching invariant unit within the chains $[14,18]$.

$\lambda_{m}$, one of the van der Waals parameters, describes the heuristical value of maximum extension of the network. In the case of chains of sufficiently large length, $\lambda_{m}$ can be related to the average length of the chains [12-18]

$$
\lambda_{m}=y_{N}^{1 / 2} \approx(\alpha\langle y\rangle)^{1 / 2} ; \alpha=M_{o} / M_{S t}
$$

where $y_{N}$ is the average number of strain invariant units per chain and $\langle y\rangle$ is the average number of monomer units per chain, with $M_{o}$ as the molecular weight of the monomer unit. $\alpha$ relates the size of the thermodynamically relevant monomer unit (for example the isoprene-unit) to the size of the stretching invariant unit. $\phi$ is the elastic potential of an incompressible isotropic network $[15,16]$

$$
\phi=\left(I_{1}-3\right) / 2=\left(\lambda^{2}+2 / \lambda-3\right) / 2
$$

with $I_{1}$ as the first strain invariant. $\eta$ is defined by

$$
\eta=\phi / \phi_{m}
$$

with

$$
\phi_{m}=\left(I_{1 m}-3\right) / 2=\left(\lambda_{m}^{2}+2 / \lambda_{m}-3\right) / 2
$$

$a$ is the second van der Waals parameter which is introduced to take into consideration "global interactions" between the network chains [12-16].

\section{Thermodynamics of eutectoid copolymers}

Referring to recent publications $[10,19]$, the thermodynamics of crystallizing networks as eutectoid copolymers is shortly recalled in this section.

The crosslinks are considered to be expelled from the crystallographically ordered crystal cores. Each single link corresponds to two non-crystallizable units. The crystallizable sequences (c-sequences) form extended-sequence mixed crystals (see fig. 1). By segregation of these sequences into mixed crystallites a crystal thickness distribution is developed which is uniquely related to the chain length distribution in the network.

The solubility within the extended-sequence crystals (EMC) is approximately described by the relation $[10,19]$

$$
\Delta y=A y+B, B=B_{1} x_{c} \mid x_{n c}+B_{o}
$$

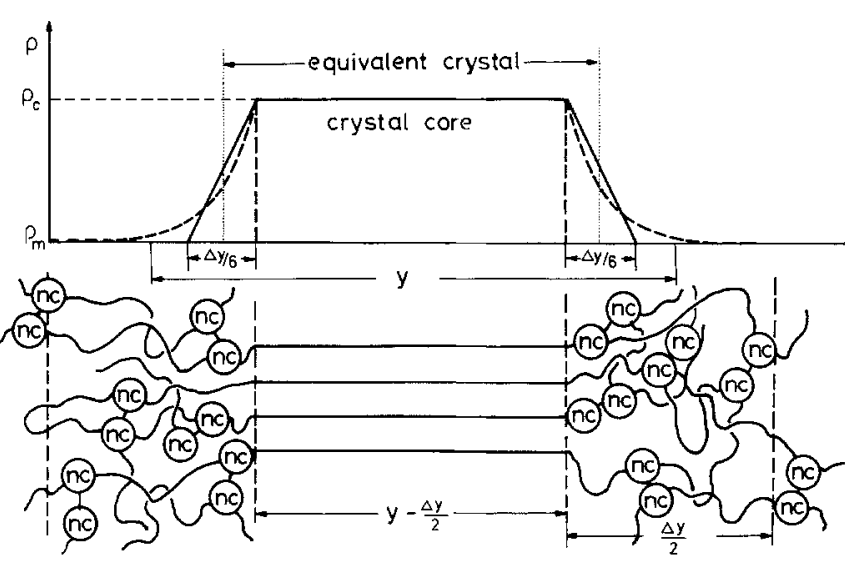

Fig. 1. Model of a eutectoid $c$-sequence mixed crystal. This is strictly seen as a non-homogenious microphase with defects as well as crosslinks regulating the disordered atomistic state of order of the longitudinal boundary layers of the average thickness $\Delta y / 2$. The solubility of the $c$-sequences of different lengths is in principle limited. Simplifying the actual situation the disparity in $c$-sequence lengths is on average characterized by $\Delta y=A y+B$ (see text). Using the equivalent two-phase model, it it is evident from the sketch that $n c$-units "are built into this crystal". The concentration of these units is uniquely dependent upon $\Delta y(y)$. The sketch in the upper part of this scheme is a drawing of the average density whereby the course in the boundaries is not exactly known. $\left(\varrho_{c}\right.$ : density of the crystal core; $\varrho_{m}$ : density of the non-crystallized amorphous regions; (20) crosslinks) 
where $A$ and $B, B_{1}$ and $B_{o}$ are constants, while $y$ is the average number of periodic units per $c$-sequence within the EMC. $x_{c}$ is the molar fraction of the crystallizable co-units while $x_{n c}$ gives the molar fraction of the non-crystallizing units $\left(x_{c}+x_{n c}=1\right)$.

The melting temperature $T_{m y}$ of the EMC characterized by $y$ was derived to be equal to

$$
T_{m y}=T_{m}\left(1-2 \sigma_{e} / N_{1}\right) / N_{2}
$$

with

$$
\begin{aligned}
N_{1}= & (1-A / 3)\left(y-y_{k}\right) \Delta h \\
N_{2}= & 1+\frac{R T_{m}}{N_{1}}\left\{\ln \left(\frac{y}{\Delta y / 2+1}\right)-\ln \left(\frac{x_{y}^{m}}{x_{y}^{c}}\right)\right. \\
& +(y-1) \chi / 2\}
\end{aligned}
$$

$T_{m}$ is the maximum melting temperature of a single component system comprised of EMCs of "infinite" thickness. The molar melting enthalpy depends upon temperature according to

$$
\Delta h(T)=\Delta h\left(T_{m}\right)-\Delta C\left(T_{m}-T\right)
$$

with $\Delta C$ as molar heat capacity difference between crystal- and meltphase. $2 \sigma_{e}$ is the longitudinal molar free surface enthalpy with a temperature dependence given by

$$
2 \sigma_{e}(T)=2 \sigma_{e}\left(T_{m}\right) \Delta h(T) / \Delta h\left(T_{m}\right)
$$

$y_{k}$ accounts for defects in the longitudinal boundaries (see fig. 1) and is defined by

$$
y_{k}=(B / 3-1 / 2) /(1-A / 3) .
$$

The mixing entropy depends upon the molar fraction $x_{y}^{c}$ of the $c$-sequences of the length $y$ within the EMC involved

$$
x_{y}^{c}=x_{n c} /\left(x_{c}^{-\Delta y / 2}-x_{c}^{\Delta y / 2}\right)
$$

and upon the conjugated molar fraction $x_{y}^{m}$ in the melt

$$
x_{y}^{m}=x_{n c} x_{c}^{y}\left(1-\varepsilon_{o}\right) /\left(1-x_{c}^{y}\right)
$$

where

$$
\varepsilon_{o}=\left(1-x_{c}^{\Delta y / 2}\right) /\left(x_{c}^{-\Delta y / 2}-x_{c}^{\Delta y / 2}\right) .
$$

The molar degree of crystallinity is then obtained to be given by

$$
w_{c}(T)=(1-A / 3) x_{c}^{(y(T)-1)}\left\{\left(y-y_{k}\right) x_{n c}+x_{c}\right\} .
$$

The average crystal thickness can be written as

$$
\left\langle y_{c}\right\rangle=(1-A / 3)\left(x_{c} / x_{n c}+y-y_{k}\right)
$$

indicating that $\left\langle y_{c}\right\rangle$ is in unique dependence on temperature. On discussing the maximum melting temperature where the last EMCs of largest average thickness are observed to dissappear, $T_{m y}$ must be computed for very small amounts of $w_{c}\left(w_{c}=0.01-0.001\right)$ such that the concentration in the multi-component melt corresponds practically to its concentration in the non-crystallized state.

The effects of raising the density of crosslinks are that the maximum melting temperature and the maximum degree of crystallinity are depressed (eutectoid system). As in each multicomponent system [19-22], definite mass-fractions of the coexisting microphases, each of them with a specified composition, are dictated by thermodynamics at each temperature in the melting range. The degree of crystallinity is thus found to be uniquely regulated in dependence of the temperature by partial selective melting of defined fractions of EMCs.

The question whether the crosslinks are randomly distributed or not can be answered by a quantitative interpretation of experimental data: excellent agreement between calculations for statistically random copolymers and experiments in case the of PE networks can be seen by evidence from figure 2 [10] such that $a$ statistically random distribution of the junctions in radia-

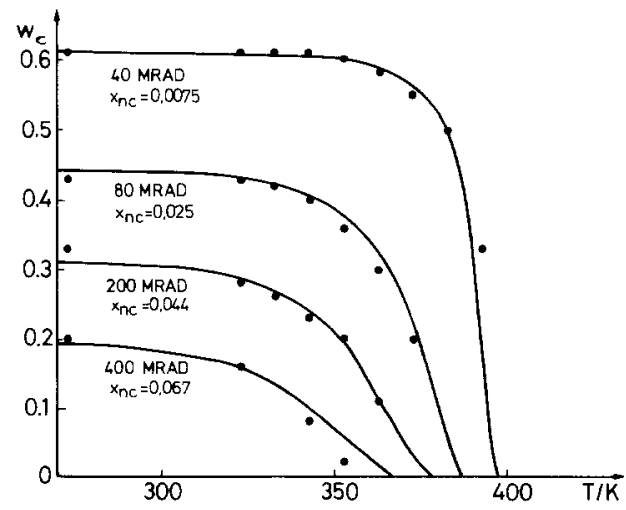

Fig. 2. Calculated molar mass fraction $\left(w_{c}\right)$ of crystals of different polyethylene networks as a function of temperature $(-)$ compared with WAX data (O), according to [10] 
tion-crosslinked PE irradiated in the molten state must be indeed assumed to be present.

\section{Oriented crystallization in the strained network}

Crystallites formed in an oriented network deformed by simple extension to $\lambda=L / L_{0}$ ( $L$ actual length, $L_{o}$ initial dimensions in direction of the stress applied), are always found to be fully oriented [23-25, $30]$. For not too short $c$-sequences there is thus a contribution to the macrosopic strain $\lambda$ given by $w_{c} \lambda_{c}$, thus yielding

$$
\lambda=w_{c} \lambda_{c}+\left(1-w_{c}\right) \lambda_{a}
$$

where $\lambda_{a}$ describes the intrinsic elongation in the noncrystallized fraction of the sample. Hence, it is assumed that the macroscopical strain $\lambda$ is obtained by contributions added according to the volume fractions of both of the micro-phases present (volume fraction $\sim$ mass fraction $\sim$ molar fraction of the crystallites as defined by $w_{c}$ ).

Crystallization is now determined by stipulating equality of the chemical potential of the $c$-sequences of the length $y$ in the $\operatorname{EMC~}(y)\left(\mu_{y}^{c}\right)$ and in the melt $\left(\mu_{y}^{m}\right)$.

Hence, for computing

$$
\left(\partial G^{c} / \partial n_{y}^{c}\right)_{T, P} \equiv \mu_{y}^{c}=\mu_{y}^{m} \equiv\left(\partial G^{m} / \partial n_{y}^{m}\right)_{T, P}
$$

with $G^{\alpha}$ as the free enthalpy of the phase $\alpha$ and $n_{y}^{\alpha}$ the corresponding molar numbers of the sequence of the length $y$, we have to know the intrinsic elongation in the oriented melt which is obtained from equation (17) to be equal to

$$
\lambda_{a}=\left(\lambda-w_{c} \lambda_{c}\right) /\left(1-w_{c}\right) .
$$

On the use of equation (2) and by introducing an empirical factor $\xi$, we are led to

$$
\lambda_{a}=\left(\lambda-w_{c} \sigma\left(\left\langle y_{c}\right\rangle\right)^{1 / 2}\right) /\left(1-w_{c}\right) ; \sigma=\xi \alpha^{1 / 2}
$$

$\xi$ should include effects related to the spatial configuration of the oriented EMCs or to situations occurring in the longitudinal boundaries both of which may systematically modify the $\lambda_{c}$ as defined in equation (2).

To perform the derivatives, we have first to define the molar strain energy function of the van der Waals network. The number of chains in the amorphous regions matters only. Hence with equation (1) we arrive at

$$
g^{m}=\sum_{y=1}^{y_{\min }(T)} n_{y}^{m} g(\lambda)
$$

with $n_{y}^{m}$ as the molar number of the $c$-sequences of length $y$ in the amorphous regions. According to equation (1) $w$ as the molar free enthalpy of deformation per chain is equal to

$$
g(\lambda)=R T\left\{-2 \phi_{m}\left(\ln \left(1-\eta^{1 / 2}\right)+\eta^{1 / 2}\right)-\frac{2}{3} a \phi^{3 / 2}\right\} .
$$

With equation (21) equipartition of energy of deformation is indeed postulated to exist: each of the chains within the network (as autonomous subsystem of deformation) is deformed such as to contribute with the same $g(\lambda)$ to the strain energy.

The partial molar free enthalpy of the $c$-sequences of length $y$ in the rubbery regions becomes

$$
\left(\partial g^{m} / \partial n_{y}^{m}\right)_{T, P}=g(\lambda)+\sum_{v} n_{v}^{m}\left(\partial g(\lambda) / \partial n_{y}^{m}\right)_{T, P} .
$$

The derivate $\left(\partial w / \partial n_{y}^{m}\right)_{T, P}$ is obtained to be given by

$$
\begin{aligned}
\sum_{\nu} n_{v}^{m}\left(\partial g / \partial n_{y}^{m}\right)_{T, P} \\
=\frac{1}{1-\eta^{1 / 2}}\left(\partial \eta / \partial n_{y}^{m}\right)_{T, P} \sum_{\nu} n_{v}^{m} \phi_{m} \\
\quad-2 \sum_{v} n_{v}^{m}\left(\frac{\partial \phi_{m}}{\partial n_{y}^{m}}\right)_{T, P}\left(\ln \left(1-\eta^{1 / 2}\right)+\eta^{1 / 2}\right) \\
\quad-a \phi^{1 / 2} \sum_{v} n_{v}^{m}\left(\partial \phi \mid \partial n_{y}^{m}\right)_{T, P} .
\end{aligned}
$$

For $\sum_{v} n_{v}^{m}\left(\partial \eta / \partial n_{y}^{m}\right)_{T, P}$ we are led to

$$
\begin{aligned}
\sum_{\nu} n_{\nu}^{m}\left(\frac{\partial \eta}{\partial n_{y}^{m}}\right)_{T, P}= & \phi_{m}^{-1}\left(\sum_{\nu} n_{v}^{m}\left(\frac{\partial \phi}{\partial n_{y}^{m}}\right)_{T, P}\right. \\
& \left.-\eta \sum_{\nu} n_{\nu}^{m}\left(\frac{\partial \phi_{m}}{\partial n_{y}^{m}}\right)_{T, P}\right)
\end{aligned}
$$

where

$$
\sum_{\nu} n_{\nu}^{m}\left(\frac{\partial \phi}{\partial n_{y}^{m}}\right)_{T, P}=\frac{\partial \phi}{\partial \lambda_{a}} \cdot \sum_{\nu} n_{\nu}^{m}\left(\frac{\partial \lambda_{a}}{\partial n_{y}^{m}}\right)_{T, P} .
$$


Here we have

$$
\frac{\partial \phi}{\partial \lambda_{a}} \equiv D=\lambda_{a}-\lambda_{a}^{-2}
$$

and

$$
\begin{aligned}
\sum_{\nu} n_{v}^{m}\left(\frac{\partial \lambda_{a}}{\partial n_{y}^{m}}\right)_{T, P} & \frac{1}{\left(1-w_{c}\right)^{2}}\left\{\lambda-\sigma\left(\left\langle y_{c}\right\rangle\right)^{1 / 2} \sum_{\nu} n_{v}^{m}\left(\frac{\partial w_{c}}{\partial n_{y}^{m}}\right)_{T, P}\right. \\
& -\left(1-w_{c}\right)\left[\sigma \sum_{\nu} n_{\nu}^{m}\left(\frac{\partial w_{c}}{\partial n_{y}^{m}}\right)_{T, P}\left(\left\langle y_{c}\right\rangle\right)^{1 / 2}\right. \\
& \left.\left.+\frac{\sigma w_{c}}{2\left(\left\langle y_{c}\right\rangle\right)^{1 / 2}} \sum_{\nu} n_{v}^{m}\left(\frac{\partial\left\langle y_{c}\right\rangle}{\partial n_{y}^{m}}\right)_{T, P}\right]\right\} .
\end{aligned}
$$

Taking into account that $d n_{y}^{m}=-d n_{y}^{c}$, the partial molar changes of $w_{c}$ are obtained from

$$
\sum_{\nu} n_{v}^{m}\left(\frac{\partial w_{c}}{\partial n_{y}^{m}}\right)_{x, P}=-y\left(1-x_{c}^{v}\right) x_{n c} \mid x_{c} .
$$

At least, the variation of the average thickness of the EMCs is defined by

$$
\sum_{\nu} n_{\nu}^{m}\left(\frac{\partial\left\langle y_{c}\right\rangle}{\partial n_{y}^{m}}\right)_{T, P}=-\sum_{\nu} n_{\nu}^{m}\left(\frac{\partial\left\langle y_{c}\right\rangle}{\partial n_{y}^{c}}\right)_{T, p} .
$$

For the melting point of the EMCs of the average thickness $y$, we then arrive at

$$
T_{m y}=T_{m}\left(1-2 \sigma_{e} / N_{1}\right) / N_{3}
$$

with

$$
N_{1}=(1-A / 3)\left(y-y_{k}\right) \Delta h(T)
$$

and

$$
\begin{aligned}
N_{3}= & +\frac{R T_{m}}{N_{1}}\left\{\ln \left(\frac{y y}{2}+1\right)-\ln \left(\frac{x_{y}^{m}}{x_{y}^{c}}\right)\right. \\
& \left.+\frac{\chi}{2}(y-1)-\frac{1}{R T}\left(\frac{\partial g^{m}}{\partial n_{y}^{m}}\right)_{T, p}\right\} .
\end{aligned}
$$

From equation (33) we realize that the partial molar entropy difference of the $c$-sequences $y$ being in the crystallites and in the melt, is continuously diminished with increased intrinsic extension in the non-crystallized regions due to the partial molar entropy term $\left(\partial g^{m} / \partial n_{y}^{m}\right)>0$.

For an understanding of the strain-induced crystallization, it is important to bear in mind that the intrinsic strain in the amorphous parts will increasingly become relaxed when oriented crystallization precedes this according to the definition $\lambda_{a}$ as given in equation (20). The "stabilizing effect" of the orientational entropy loss in the non-crystallized regions is thus reduced when oriented crystallization advances.

Only a comparison with experiments delivers a proof of the utility of the thermodynamic relations presented above. We should also ask how essential structure parameters in the semicrystalline system with fully oriented crystallites are correctly described by the theory presented. A defined crystallite thickness distribution is predicted showing specified dependencies upon the concentration of crosslinks, the macroscopic strain and the temperature as well. A first characterization can be given by discussing the average thickness of the EMCs and the total amount of existing crystallites described by the molar degree of crystallinity. On the use of both of these structure parameters, the following predictions can be made:

a) The degree of crystallinity is systematically reduced when the density of crosslinks is increased.

b) There is on principle a melting range the topological characteristics of which is determined by the network chain-length distribution with its concomitant influence on the crystal thickness distribution. By selective partial melting of just the smallest EMCs, the width of the crystal thickness distribution is continuously diminished accompanied by a corresponding increase of its average thickness $\left\langle y_{c}\right\rangle$.

c) By simple extension the melting temperature of the $E M C$ is shifted to highervalues when compared with the situation in the non-oriented network. The shift of the melting temperatures is largest for the smallest crystals being systematicly diminished with increasing thickness of the EMCs. Hence, the melting range is predicted to become smaller in oriented systems, the more the higher the total degree of extension $(\lambda)$.

d) Effects due to finite length of the chains as well as due to global interactions between the chains (as considered with the van der Waals strain energy function), are manifested by typical features of strain-induced crystallization: it is for example in principle possible to bring the network to its maximum degree of crystallinity at temperatures above the maximum $T_{m}$ by going to sufficiently large macroscopical extensions such that 
the upturn in the stress-strain curve due to finite chain extensibility comes into play.

e) The concepts presented are only appropriate as long as extended $c$-sequence mixed crystals are formed. If the density of the crosslinks is reduced below a certain critical concentration, folded chain crystallization occurs bringing about a different situation which implicates dependence of kinetical conditions of crystallization.

\section{Comparison with experiments}

The quality of the fit of calculations for natural rubber with the aid equations (31)-(33) is seen by evidence from the plot in figure 3 . The data used are listed in table 1 . The molar enthalpy $\Delta h\left(T_{m}\right)$ per monomer unit corresponds approximately to the data as given by van Krevelen [26]. In correspondence to polyethylene [27], the molar surface enthalpy was computed with the aid of the evident relation $\sigma_{e}\left(T_{m}\right) \cong \Delta h\left(T_{m}\right)$. The melting temperature $T_{m}$ is assigned to the value as indicated in table 1 whereby that value was accepted by which the best fit to the experimental stress-strain curves of natural rubber was obtained. The value of $T_{m}$

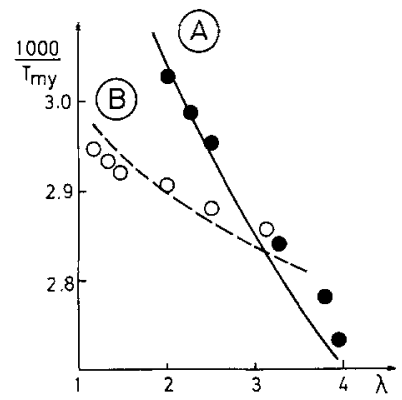

Fig. 3. Plot of the reciprocal maximum melting temperatures against macroscopic strain: (A) natural rubber: experimental data according to [6], - calculated using the parameter listed in table 1; (B) Polychloroprene rubber: $O$ experimental data according to $[6],---$ computed with the use of the parameter compiled in table 2

Table 1. Natural rubber

$\begin{array}{ll}\Delta h\left(T_{m}\right) & =3100 \mathrm{cal} / \text { mole units } \\ 2 \sigma_{e}\left(T_{m}\right) & =6200 \mathrm{cal} / \text { mole units surface } \\ T_{m} & =295 \mathrm{~K} \\ \lambda_{m} & =9.8 \\ x_{c} & \left.=0.97^{\mathrm{a}}\right) \\ \chi & =-0.3 \\ B_{1} & =0.9 \\ \Delta C & =1.4 \mathrm{cal} / \mathrm{mole} \text { degree unit } \\ \alpha & =3 \\ \xi & =0.63 \\ B_{o} & =70\end{array}$

$$
\text { a) } \lambda_{m}^{2}=\alpha\langle y\rangle ; x_{c}=\langle y\rangle /(\langle y\rangle+1)
$$

under discussion is lower than data reported in literature [26]. We leave this discrepancy to a discussion in future publications.

We should like now to estimate the actual size of $\lambda_{m \mathrm{Cl}}$, the maximum strain in chloroprene-networks. From the representation of stress-strain measurements on polychloroprene [16], a modulus was obtained

$$
G_{\mathrm{Cl}}=\varrho_{\mathrm{Cl}} R T /\left(\lambda_{m \mathrm{Cl}}^{2} M_{S t \mathrm{Cl}}\right)=0.595 \mathrm{MPa}
$$

where $M_{S t \mathrm{Cl}}$ is the molecular weight of the stretching invariant chain unit in the polychloroprene network. The above value will now be compared with the modulus of natural rubber of [16]

$$
G_{R}=\varrho_{R} R T /\left(\lambda_{m R}^{2} M_{S t R}\right)=0.359 \mathrm{MPa}
$$

where $\lambda_{m R}=10$ was obtained from the fit of calculations to the "reduced" force $f / G_{R}[16]$. Provided that the stretching invariant units have the molecular weights

$$
\begin{aligned}
& M_{S t R}=68 \mathrm{~g} / \mathrm{mol} \alpha^{-1} \text { (natural rubber) } \\
& M_{S t \mathrm{Cl}}=90 \mathrm{~g} / \mathrm{mol} \alpha^{-1} \text { (polychloroprene) }
\end{aligned}
$$

and the densities are given by [28]

$$
\begin{aligned}
& \varrho_{R}=980 \mathrm{~kg} / \mathrm{m}^{3} \text { (natural rubber) } \\
& \varrho_{\mathrm{Cl}}=1300 \mathrm{~kg} / \mathrm{m}^{3} \text { (polychloroprene) }
\end{aligned}
$$

we may thus calculate $\lambda_{m \mathrm{Cl}}$ of the chloroprene network with the aid of the relation

$$
\lambda_{m \mathrm{Cl}}^{2}=\left(\varrho_{\mathrm{Cl}} M_{S t R} G_{\mathrm{Cl}} / \varrho_{R} M_{S t \mathrm{Cl}} G_{R}\right) \lambda_{m R}^{2}
$$

With the data as given in the present case, we are led to $\lambda_{m a}=12.9$. The other parameters used $\left(\Delta h\left(T_{m}\right)\right.$, $\left.2 \sigma_{e}\left(T_{m}\right), \Delta C, T_{m}\right)$ are compiled in table 2 . The fit of

Table 2. Chloroprene network

\begin{tabular}{ll}
\hline$\Delta h\left(T_{m}\right)$ & $=6666 \mathrm{cal} /$ mole units \\
$2 \sigma_{e}\left(T_{m}\right)$ & $=13332 \mathrm{cal} /$ mole units surface \\
$T_{m}$ & $=320 \mathrm{~K}$ \\
$\lambda_{m}$ & $=13$ \\
$x_{c}$ & $\left.=0.983^{\mathrm{a}}\right)$ \\
$\chi$ & $=-0.15$ \\
$B_{1}$ & $=0.9$ \\
$\Delta C$ & $=1.4 \mathrm{cal} /$ mole degree unit \\
$\alpha$ & $=3$ \\
$\xi$ & $=0.63$ \\
$B_{o}$ & $=70$
\end{tabular}

$\left.{ }^{a}\right)$ Calculated with the relation as given with table 1 


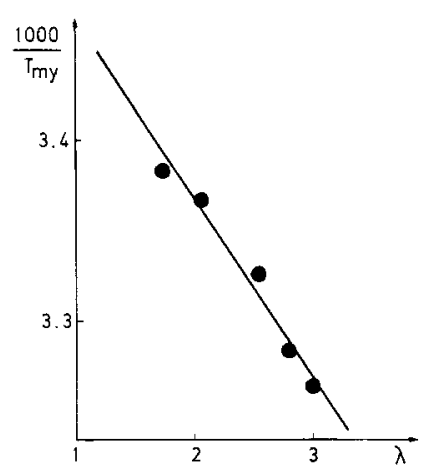

Fig. 4. Maximum melting temperatures in natural rubber with a low degree of crosslinking. measured [6]; —- interpolated

calculated $T_{m y}^{-1}(\lambda)$ to the experimental data is also drawn out in figure 3 . For natural rubber with a comparatively low concentration of crosslinks (see fig. 4) melting temperature calculations could not be fitted to the data at all. A possible source of systematical errors in this case may be related to the methods of determining the "maximum" melting temperatures. Other reasons behind that failure might also be given by nonhomogeneity of this weakly crosslinked network or by the appearance of folded-chain crystals with their typical dependence upon crystallization history.

\section{Discussion}

The good accord between calculations and experimental results defends the basic assumption that straininduced crystallization of macromolecular networks should be interpreted as crystallization of a entectoid copolymer systems, from an oriented equilibrium melt. It is important to note that the solubility within the extended $c$-sequence mixed crystals seems not to be altered at all such that the EMCs can indeed be considered as "thermodynamically autonomous mixed non-homogeneous micro-phases".

At the maximum melting temperature we bave therefore at the various states of deformation the same degree of crystallinity always showing the same assembly of fully oriented crystallites. The shift of the maximum melting points of a network is thus determined by the partial molar entropy of deformation of the chains $y$ brought into states of different orientation by simple extension (having basically retained the liquid-like short range order [14-16]). The state of deformation in the rubbery melt is fairly well described by means of the van der Waals approach. Moreover, due to having always achieved internal equilibrium [19], the strain-energy in the melt is governed by its global state of orientation only. Because of having energetically equivalent chains each of them contributes to the entropy of deformation (in spite of being linked within the molecular network) the same amount of partial molar entropy of deformation.

In that concern, we find it very satisfactory that strain-induced crystallization in chemically different networks like natural rubber and polychloroprene networks are found to be interpreted with the same theory. The difference in the strain-induced shift of the maximum melting point to higher temperatures between natural rubber and polychloropren networks (see fig. 3), can be related to two origins:

1. The relatively large enthalpy of melting of the chloroprene crystals reduces the influence of the orientational entropy within the rubbery amorphous areas (see eqs. (31) $-(33)$ ).

2. The liquid-like short range structure within both networks under discussion differs, which is phenomenologically expressed by different values of the $\chi^{-}$ parameters.

It might be interesting to stress that these individual features in the $T_{m y}^{-1}(y)$-dependence must indeed be known for the quantitative representation of the experimental data as given in figure 3. A discussion on these lines is in fact possible only because of the analogue global properties of van der Waals networks.

\section{Parameters of the micro-structure}

It is now of the utmost importance to prove whether essential "model parameters" of the eutectoid systems characterizing the semicrystalline structure in oriented networks can be verified by experimental evidence.

First we prove the question whether the average thickness of the $c$-sequence mixed crystals is correctly predicted with the aid of the present theory. It was found by one of the authors from electron microscopical investigations of gold-stained samples of natural rubber that the average thickness of the EMCs at room temperature should be equal to $\left\langle y_{c}^{*}\right\rangle \cong 15 \mathrm{~nm}[30,31]$.

For comparing this datum with calculations we have to decide which "part of the EMCs" (see fig. 1) can in fact be seen in the electron microscopic pictures. It is a straightforward assumption to assume that only the well ordered core of the EMCs will not be stained at all. In that case, we can easily compute the average value in the polydispersed thickness distribution of the EMCs at room temperature for strained samples. The distorted layer of the longitudinal layers is of the aver- 
age thickness $\Delta y / 2$, thus, yielding for the average thickness of the crystallograficly ordered core (see fig. 1):

$$
\left\langle y_{c}^{*}\right\rangle=x_{c} / x_{n c}+y_{\min }-\Delta y / 2
$$

With $\alpha=3$ for $\lambda=6$, we are then led to the value of

$$
\left\langle y_{c}^{*}\right\rangle=17 \mathrm{~nm}
$$

where $x_{c}=0.97, B_{o}=70$ and $y_{\min }=50$ units.

This value is very close to the electron-microscopic observation thus defending the concept of baving the crystal thickness distribution of the EMCs (and also their average thickness) uniquely determined by the chemical structure of the network.

In that case, it should also be feasible to compute the molar degree of crystallinity in largely strained systems of natural rubber. The degree of crystallinity determined by WAXS methods is also expected to give the mass fraction of the crystallographically ordered crystal cores only. This can be justified with the theory of paracrystals [29]. In this case, the molar fraction of the crystal cores can be computed with the aid of the relation

$$
w_{c o}=x_{c}^{y-1}\left\{(y(1-A / 2)-B / 2) x_{n c}+(1-A / 2) x_{c}\right\} .
$$

For the natural rubber $\left(x_{c}=0.97, y_{\min }=50\right.$ units, $A=$ $0.15, B_{1}=0.9, B_{0}=70$ ) at room temperature extended to $\lambda=6$, we arrive at $w_{c o}=0.19$ a value which compares fairly well with the experimental value of 0.18 [32]. It should be notified that the "actual two-phase degree of crystallinity" of the EMCs in the network is obtained to be equal to $w_{c}=0.29$. The above correspondence is indeed of essential importance: it verifies the basic assumption that the junctions are "non-crystallizable", such that their molar fraction determines that maximum degree of crystallinity which can be attained at all.

\section{Concluding remarks}

The quantitative reperesentation of the experimental data under discussion allows - in spite of being in need of more systematic experiments - to summarize the following conclusions:

- The network behaves like a weakly interacting van der Waals conformational gas represented by an analytically defined strain-energy function. Finite chain length and global interactions regulate the strain energy stored on deformation.
- The oriented c-sequence extended chain crystals are found to be basicly identical with the crystals observed for crystallization in the quasi-isotropic state. Hence, the EMCs can be considered to represent "thermodynamically autonomous non-homogeneous micro-phases" as essential "bricks" constituting in dependence on the size distribution a complicated colloid-structure.

- The maximum degree of crystallinity is always in unique relationship to the molar fraction of crosslinks. The junctions are expelled from the well ordered crystal cores.

- The apperance of the EMCs do not modify the effective degree of crosslinking. For thermodynamic reasons, the extended $c$-sequence mixed crystals have always to adjust on deformation their intrinsic concentration and their mass fraction. These rearrangements are the very important reason behind the observation of not having relevant "active crosslinking effects" by strain-induced crystallites.

Because of having the EMCs fully oriented, the intrinsic elongation within the rubbery region is reduced in dependence on the average crystal thickness and the mass fraction of the EMCs present.

-The chemical structure of different rubbers affects primarily the intrinsic properties of the microphases. It is significant that the topological features of extended $c^{-}$ sequence crystals remain unaltered in both cases under discussion (natural rubber and polychloroprene rubber): the solubility in the solid microphases is found to be identical. Together with the universal features of the van der Waals network, the molar melting enthalpy, the molar surface enthalpy and the $\chi$-parameter are left for describing the different $T_{m y}^{-1}(\lambda)$-dependence of both of the rubbers under discussion (natural rubber and polychloroprene).

- If the concentration of the crosslinks is small, our representation must fail from various reasons: foldedchain crystallization, non-homogeneity of the networks (sol fraction of smaller molecules!), chain-end effects and also possible differences in the melt-structure [33] violate alltogether relevant suppositions of the thermodynamic treatment of eutectoid copolymer networks.

There is no doubt, that an investigation of the mechanical, thermo-mechanical and caloric properties of the total stretching process is needed to check critically the utility of the thermodynamic theory of strained eutectoid networks as presented in this paper.

\footnotetext{
Acknowledgement

We should like to thank the AIF and the Deutsche Kautschuk Gesellschaft for financial aid.
} 


\section{References}

1. Flory PJ (1947) J Chem Phys 15:3976

2. Gaylord RJ (1976) J Polym Sci, Polym Phys Ed 14:1827

3. Gaylord RJ (1975) Polym Letters Ed 13:377

4. Kim HG, Mandelkern L (1968) J Polym Sci A 2, Vol 16:181

5. Krigbaum WR, Roe RJ (1964) J Polym Sci A 2:4319

6. Krigbaum WR, Dawkins JV, Via HG, Balta YG (1966) J Polym Sci A 2:475

7. Oth JF, Flory PJ (1958) J Am Chem Soc 80:1297

8. Mandelkern L (1964) Crystallization of Polymers, McGrawHill, New York

9. Gent AN (1954) Trans Faraday Soc 50:521

10. Heise B, Kilian HG, Schmidt H (1981) Coll \& Polym Sci 259:611

11. Kilian HG, Unseld K, Jaeger E, Jungnickel B, Mueller J (1985) Coll \& Polym Sci, in press

12. Kilian HG (1984) International Rubber Conference Moscow

13. Enderle HF, Kilian HG, Vilgis T (1984) Coll \& Polym Sci 262:696

14. Kilian HG (1981) Polymer 22:209

15. Kilian HG (1982) Coll \& Polym Sci 260:895

16. Eisele U, Heise B, Kilian HG, Pietralla M(1981) Angew Makromol Chemic 100:67

17. Treloar LRG (1958) The Physics of Rubber Elasticity, 2nd Ed, Clarendon Press, Oxford

18. Green AE, Atkins JE (1970) Large Elastic Deformations, ZdE, Clarendon Press, Oxford

19. Kilian HG, Glenz W, Klattenhoff D, Stracke F (1977) Polymer 18:685

20. Callen HB (1960) Thermodynamics, Wiley Int Ed, New York
21. Haase R (1956) Thermodynamik der Mischphasen, Springer, Berlin, Göttingen, Heidelberg

22. Kilian HG (1979) Makromol Chem, Suppl 3:277

23. Andrews EH (1964) Prog Roy Soc, London A 277:562

24. Andrews EH (1966) J Polym Sci 4:668

25. Bunn CW (1942) Proc Roy Soc, London A 180:40

26. van Krevelen DW (1974) Properties of Polymers, Elsevier Publishing Company, Amsterdam

27. Illers $\mathrm{KH}$, Hendus $\mathrm{H}$ (1968) Makromol Chemie 113:1

28. Brandrup J, Immergut EH (1975) Polymer Handbook, J Wiley \& Sons, New York

29. Hosemann R, Bagchi SN (1962) Direct Analysis of Diffraction by Matter, North Holland Publ Comp, Amsterdam

30. Hardin IR, Yeh GSY (1973) J Macromol Sci Phys B 7(2):375 and 393

31. Luch D, Yeh GSY (1972) J Appl Phys 43:4326

32. Roberts R, Mandelkern L (1955) J Am Chem Soc 77:81

33. Holl B, Heise, Kilian HG (1983) Coll \& Polym Sci 261:978

Received September 5, 1984; accepted October 5, 1984

Authors' address:

B. Holl

Universität Ulm

Abt. Experimentelle Pysik

Oberer Eselsberg

D-7900 Ulm 